\title{
Securing Heterogeneous Privacy Protection in Social Network Records based Encryption Scheme
}

\author{
${ }^{1}$ Balamurugan.R, ${ }^{2}$ Dhivakar. M, ${ }^{3}$ Muruganantham.G, ${ }^{4}$ Ramprakash.S \\ Department of Computer Science and Engineering \\ University College of Engineering - Thirukkuvalai - 610204. \\ Email ID: vijayamuruga98@gmail.com
}

\begin{abstract}
This survey places of interest the major issues concerning privacy and security in online social networks. Firstly, we discuss investigate that aims to protect user data from the an assortment of attack vantage points together with other users, advertisers, third party request developers, and the online social arrangement provider itself. Next we cover social network supposition of user attributes, locate hubs, and link prediction. Because online social networks are so saturated with sensitive information, network inference plays a major privacy role. Social Networking sites go upwards since of all these reasons. In recent years indicates that for many people they are now the mainstream communication knowledge. Social networking sites come under few of the most frequently browsed categories websites in the world. Nevertheless Social Networking sites are also vulnerable to various problems threats and attacks such as revelation of information, identity thefts etc. Privacy practice in social networking sites often appear convoluted as in sequence sharing stands in discord with the need to reduce disclosure-related abuses. Facebook is one such most popular and widely used Social Networking sites which have its own healthy set of Privacy policy.
\end{abstract}

Keywords: Privacy and Security, Online Social Network, Sensitive Information, Network Inference, Information Sharing.

$* * * * *$

\section{INTRODUCTION}

A social networking overhaul is an online service, display place, or site that focuses on fascinating the construction of social relations among people who, for example, behavior, share interests, background, or real-life connections [7]. Improvement on Online Social Networks the user can connect with their acquaintances very easily. They can share data or view movies, Videos of each other. Because of all these reason growth of SNS in recent years indicates that they are now mainstream communications knowledge for many people [8]. The people who are using social network sites see them as fun and easy spare time activities. By ornamental their social circle, users have the opportunity to commune with people who have the same welfare. In today's state of affairs Social networking site are most widely used websites in world, with Face book being the succeeding most visit site on internet world wide first being Google. As on July 2013, Face book has more than 1.20 billion active users [1] and the revenue generate by Face book alone is 3.91(approx) million dollars [1]. Facebook is good for attractive consumers and consequently gaining valuable impetus of oral announcement.

The main way to get concerned is to make a fan page that offers impressive valuable to the consumer like appealing group conversations, tools or casual games for expediency. The end-goal for gaining oral announcement value is to get consumers to "like" your fan page. When they do, it does announce on their feed to all their friends and the fan gets regular update from the brand. While brand can do much more on the Facebook display place than on Twitter and YouTube. In the end most triumphant initiatives have been driven by promotions, similar to Twitter. Here privacy is an imperativeownership. We find it difficult to stifle our curiosity about others [10].Today communal networking site is just not an amusement websites, but one of the most important announcement medium in today's world. Nonetheless there have been always a privacy and secrecy concerns about misuse the crucial information by internet perpetrators. Also Social network site are a perfect platform for virus authors to spread their malwares faster than long-established methods. Facebook scams in recent time's hits the headlines in the Internet scam world.

\section{PROTECTING USER DATA}

In any online social set of connections users are generating a massive quantity of data. In this section we focal point on data the users create on purpose with the objective of distribution (i.e., explicit information). This includes blog or micro-blog posts, profile in rank, photos, videos, instant communication text, and so on. This does not include data such as schmaltzy relationships with other users, or account construction times. We refer to this type of unambiguous, "for sharing" data as "content." There are several dissimilar vantage points from which an adversary cansadmittance user data in aup-to-the-minute online social network. Supplementary users are a constant threat because in the vast majority of OSNs today anybody (including malevolent parties) can sign up for an explanation and become a member thereby ever-increasing their access to 
other users' data. The only necessities are a valid email address and the ability to solve a re-captcha. This means the social set of connections provider must provide the users some way to spell out who they trust and who they don't trust within the social network. Different providers handle this in dissimilar ways with varying levels of detail. Social network "applications" are web pages that are in black and white by some third party but have API right of entry to social graph data typically only accessible by the online social network provider. Application are typically opt-in for users. In the case of Facebook users are told plainly and vaguely what content an application will attempt to access and are prearranged the choice whether or not to use that submission.

\subsection{Protection from Other Users}

Protecting users from "other users" include any other user on the social system. We can partition the set of other user into three categories.

\section{Directly Connected Users}

These are users that have a link flanked by them in the community graph. This means something different in poles apart social networks. In Facebook it means that the two users can view more in sequence on each other's profile. In some of the literature this simply income the two users have communicate with every one other via email.

\section{Unconnected or Indirectly Connected Users}

These users are two or supplementary hops away from one an additional. (e.g., friends of friends (FOF) or acquaintances of associates of friends (FOFOF)). This category also includes two users that are in the online social network that have completely no relative between them.

\section{General Public}

The wide-ranging public has access to in succession in many online social networks. For example, twitter makes chirrup public by default and Google index them.

\subsubsection{Direct Access}

Many online social network providers allow the users of their social set of connections to make privacy surroundings. This is the user's first line of defense adjacent to malicious users. Some of these privacy surroundings schemes are simple and undemanding. For illustration, Twitter agree to users to make tweets "confidential" which are only visible to their followers. This is the only time alone setting they make available and it efficiently locks out the general community and independent users. However, most online social set of connections providers like Facebook and LinkedIN subject their users to a dizzying, multifaceted set of privacy controls. The repercussions of the decision users are forced to make straight away are not fully recognized until the user is recognizable with the online social network in question. These time alone settings must be carefully weighed and experimentation with, and yet users are forced to make privacy setting immediately upon amalgamation the social network in order for them to be triumphant in suspicious their own data.

\subsubsection{Indirect Access}

One of the more understated issues in defensive user data from other users is the increase of sensitive, to some extentconfidential content. The key difference flanked by this and the preceding section is malevolent users access other user content straight vs. circuitously. A malicious user accesses in sequence indirectly when some third party user spreads that in sequence. Social networks characteristically try to define some set of rules for the user to define who can view their in sequence and who cannot. Anybody, however, is allowed to publish in sequence. The problem with this is that users that have access to the susceptible, hidden data of another user can basically use their ability to publish to spread that data to users whom are not hypothetical to have access to it. This can be thought of intuitively as telling a clandestine. One user knows the secret and may tell select other users that secret. However, any one of the less important users have the ability to spread the secret further, possibly to people that were never proposed to hear the message in the first place. This topic is referred to in the rest of this paper as "leaking." Many social networks unintentionally encourage this behavior by providing a builtin instrument for propagating content. A good example of this is Twitter's re-tweet functionality. Conversely, social networks that effort to prohibit users from spreading secluded content in this way are only fooling themselves. Any user that is allowed to read and allowed to bring out will have the ability to spread susceptible information. At the very least, they can read the content from one browser window and re-type it into a succeeding.

\subsubsection{Protection from the OSN Provider}

Very recently much work has gone into defensive user content from the online social network provider. The online social network supplier acts as the "Eye of God" in that it can see all data that flows through the set of connections. Currently users sign privacy policies and terms of use agreement with the supplier which is their only line of defense. Users are now commencement to realize that they do not want to trust online social network provider with their personal data. In an online social network in the client / 
server architecture that does not rely on the OSN provider to be trusted. in its place the server simply provides availability. That is, name resolution of members in the social arrangement. The actual content of the social network resides on individuals' computers multiply across the Internet. Visualize two users bobble and Alice want to communicate via the online social network. Alice's computer needs to connect to Bob's supercomputer but she does not know Bob's IP address and he does not have a sphere of influence name. In fact, it is very likely that both Bob and Alice were given self-motivated IP addresses by their ISPs and that there machines are following routers. In order to avoid elaborate configuration necessary for every member of the OSN we instead rely on the OSN servers only for name declaration.

\section{PROPOSED APPROACH}

\subsection{Group-Oriented Convergence Cryptosystem (GCC)}

To protect receptive information in web services from not permittedaccess is to encrypt in sequence using user-controlled keys and to provide access to data using user-controlled delegation. This approach is constructing on a new group-oriented junction cryptosystem (GCC), which apparatusencryption and substantiation for groups.In this project, a hierarchical admittance control method using a Modified Hierarchical Attribute-Based Encryption (M$\mathrm{HABE}$ ) and a customized three-layer structure is projected in online social network. Differing from the existing paradigms such as the HABE algorithm and the original three-layer structure, the novel proposal mainly focuses on the data processing, store and accessing.It is designed to ensure the submission users with legal access authorities to get corresponding sensing data and to restrict illegal users and not permitted legal users get access to the data; the proposed promising paradigm makes it particularly suitable for the mobile cloud computing based hypothesis. The most striking characteristic of this cryptosystem is that this system is organized and managed in a spontaneous way without a system administrator. That is, a group of trusted users, not one user, collaborate to manage and maintain a private group of people. Moreover, this cryptosystem does not need a PKC/PKI system to realize the switch over of group key.

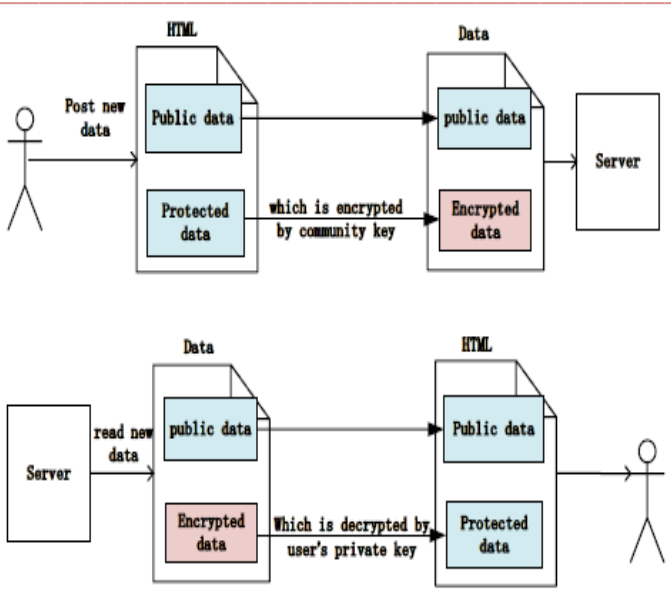

Figure 1.1 Community creation in OSN

To use GCC, each consumer in OSN generates the user's confidential key by him and registers a public label into the OSN. To create a community, some known users with the same interest (called as the creators of population) generate a community key (CK) in anassistance way. All of the creators' confidential keys are valid for this population key. For each acquaintance, a user can then generate an access permission key (APK) corresponding to his own private key and the friend's public sticky tag. Using the confidential key and the community's APK, the user can decrypt (or right of entry) the shared information, but not encrypt (or publish) the information into the population. The encryption procedure cannot be implemented unless a user holds the neighborhood key.

In order to avoid the acceptance of $\mathrm{PKC} / \mathrm{PKI}$ systems, aimpermanent public key generated from a user's private key can be used to realize the switch over of encrypted key. In addition, there exists an efficient verification protocol, by which an untrusted storage service provider (SSP) can check whether or not a user belong to a certain community. Furthermore, in our model each user in OSN has only one secretive key. Each time the user joins in a community, she will be assign an APK key from her friends, but this APK is unacceptable for other users. This approach can successfully prevent security problems cause by the loss of access authorization key.

\subsection{Benefits:}

- $\quad$ Access charge of issue deals with providing access to sanctioned users and prevent unauthorized users to access data.

- Attaching a list of sanctioned users to each data is the simplest solution to achieve access control.

- It is an effectual, fast, and robust replica detection method specifically for mobile sensor networks. 
- That can make decision quickly and accurately.

\subsection{HABE - Encryption}

Fine-grained access manage over the outsourced cipher texts will provide more isolation protection in OSN. $\mathrm{ABE}$ can be confidential into two categories (KP-ABE) and (CP-ABE).In KP-ABE, each cipher text is label by the encryption with a set of descriptive characteristic $\mathrm{CP}-\mathrm{ABE}$ is similar to $\mathrm{KP}-\mathrm{ABE}$, except that the admittance policy is labeled with each cipher text and a secret key is connected with a user's characteristic. The main goal of the HABE primordial is to provide appropriate delegation machinery for the motivating application scenario, as well as the flexible encryption of $\mathrm{ABE}$. Admin use the practice to encrypt user credential

\section{TECHNIQUE}

- The encrypted official document will be stored in the database

- Secret key is produce to each user

- User uses the clandestine key to store the credentials

- It protects susceptible information

\section{CONCLUSION}

In this work, it commences a scheme where resources are shared among communities, which resources onlymembers of a community have access to its resources. By adopt community key administration, one can ableto keep users' possessionsnot to be disclosed, even towards the system manager. In our construction, a random sessionkey is used and encapsulated for each encryption, and only members can derive the session key and decrypt itproperly. Our proof-of-concept prototype clearly established that our scheme is practical to OSNs, allowingus to generate community keys with the convenient computation overhead. This algorithm takes ode advantage of topological and transmission redundancies and utilizes feedback, exchange only between the two communicating end-nodes. Very secure the in sequencecommunication Provide the planned System. The Project proposed a HABE, by taking advantages of hierarchical characteristic based encryption (HABE) access are in command of processing. A hierarchical access control method using a personalized hierarchical attribute-based encryption (M-HABE) and a personalized three-layer configuration is projected.

\section{REFERENCES}

R. Leenes, "Context is everything sociality and privacy in online social network sites," in Privacy and Identity
Management for Life. Berlin, Germany: Springer, 2010, pp. $48-65$.

[2] L. A. Cutillo, R. Molva, and T. Strufe, "On the security and feasibility of Safebook: A distributed privacy-preserving online social network," in Privacy and Identity Management for Life. Berlin, Germany: Springer, 2010, pp. 86-101.

[3] L. Kevin, K. Jason, and C. Nicholas, "The taste for privacy: An analysis of college student privacy settings in an online social network," J. Comput.-Mediated Commun., vol. 14, no. 1, pp. 79-100, 2008.

[4] L. A. Cutillo, R. Molva, and T. Strufe, "Safebook: A privacy-preserving online social network leveraging on real-life trust," IEEE Commun. Mag., vol. 47, no. 12, pp. 94-101, Dec. 2009.

[5] L. Gyarmati and T. A. Trinh, "Measuring user behavior in online social networks," IEEE Netw., vol. 24, no. 5, pp. 2631, Sep. 2010.

[6] J. Bollen, H. Mao, and X. Zeng, "Twitter mood predicts the stock market,” J. Comput.Sci., vol. 2, no. 1, pp. 1-8, 2011.

[7] N. Li, N. Zhang, and S. Das, "Preserving relation privacy in online social network data," IEEE Internet Comput., vol. 15, no. 3, pp. 35-42, May 2011.

[8] X. Chen and K. Michael, "Privacy issues and solutions in social network sites," IEEE Technol. Soc. Mag., vol. 31, no. 4, pp. 43-53, Dec. 2012.

[9] M. Gharibpoor and S. M. Allameh, "Online social network," IEEE Trans. Knowl. Data Eng., vol. 25, no. 3, pp. 662-676, Sep. 2013.

[10] R. Van Noorden, "Online collaboration: Scientists and the social network," Nature News, vol. 512, no. 7513, pp. 126 129, 2014

[11] B. Jiang, M. Xie, U. Topaloglu, T. Hudson, H. Eswaran, and W. Hogan, "Social network analysis of biomedical research collaboration networks in a CTSA institution," J. Biomed.Inform., vol. 52, no. 15, pp. 130-140, 2014.

[12] D. Amar, H. Jordan, and S. P. Borgatti, "Leadership in neurology: A social network analysis," Ann. Neurol., vol. 75, no. 3, pp. 342-350, 2014.

[13] G. Kumar and K. Kumar, "Network security-An updated perspective," Syst. Sci. Control Eng., Open Access J., vol. 2, no. 1, pp. 325-334, 2014.

[14] T.-W. Chiou, S.-C.Tsai, and Y.-B. Lin, "Network security management with traffic pattern clustering," Soft Comput. vol. 18, no. 9, pp. 1757-1770, 2014.

[15] C.-H. Liu, J.-S.Wang, C.-C.Peng, and J. Z. Shyu, "Evaluating and selecting the biometrics in network security," Secur.Commun.Netw., vol. 8, no. 5, pp. 727-739, 2014. 